\title{
DECIMA MESA REDONDA
}

Doctor Rafael Ramírez Merchán: -Preguntan por qué no se ha hecho la integración de la Cátedra de Obstetricia y Ginecología de la Facultad de Medicina de la Universidad Nacional, y la respuesta que debo dar como jefe del departamento es que la integración sí se está haciendo. En primer lugar, existe ya una conciencia formada en tal sentido; en segundo lugar, nadie discute actualmente su conveniencia; en tercer lugar tenemos los programas, y en general, las labores docentes integrados, tropezamos con las dificultades locativas porque están separados los dos servicios; pero ya se ha hecho la propuesta para fusionarlos.

Que quedan profesores que en su ejercicio privado se dedican preferencialmente a una de las dos ramas es un hecho reconocido; pero eso no afecta la integración en lo esencial. A medida que se vayan cumpliendo los actuales programas se irá formando el personal que constituirá el equipo docente idealmente integrado.

Doctor J. del C. Acosta: - Hace tiempo que dejé las cátedras; sin embargo, no he perdido mis conexiones con el hospital, y estoy más o menos completamente enterado de la forma en que se está llevando la enseñanza. For- mado en escuelas muy anteriores y con especialistas de cada rama por separado, en el curso del tiempo se ve que la integración la hace uno mismo, a medida que se la va exigiendo la misma clientela en la práctica. Se me hace que la integración, llevada a cabo en forma ordenada y metódica, es muy conveniente, no para obtener de una vez especialistas, cosa imposible. Encuentro criticable en casi todas las exposiciones que se han hecho en donde se está haciendo la integración, que la enseñanza se está haciendo en un tiempo absolutamente insuficiente. Yo no creo que se pueda formar a un señor, para que salga con una preparación obstétrica, en seis semanas, ni en dos meses; de la misma manera que no creo se pueda hacer lo mismo en Ginecología. La propedéutica de una y otra de estas especialidades requiere un tiempo mucho mayor, minuciosidad en la exposición y adiestramiento de los estudiantes. Veo también otro inconveniente, especialmente cuando se suprime la enseñanza teórica de estas materias, y es que el alumno aprende lo que se le alcanza a mostrar en una clínica en un transcurso de mes y medio a dos meses. Todos sabemos que hay épocas en que por una $u$ otra circunstancia, determinadas entidades patoló- 
gicas no llegan al hospital; es mucho más probable que en un período más largo sí se presenten, pudiéndoseles presentar a los alumnos, dándoles de esta manera una formación más completa. Acepto pues la integración, pero no puedo aceptar el tiempo en que se está hacienda la enseñanza, porque no lo puedo entender. Anteriormente la enseñanza de Obstetricia en la Facultad de la Universidad Nacional requería dos años; un primer año, de teoría, y un segundo año de clínica obstétrica. Hoy en día se suprimen el curso de la fisiología del embarazo y el de nociones teóricas de la patología gravídica, y se hace la clínica y la obstetricia teórica y práctica en dos meses; se me hace absolutamente absurdo e imposible.

Doctor López Escobar: - Hecha como está la pregunta, creo que debemos contestar que sí está hecha la integración. Principio tienen las cosas. Cuando acabamos de nacer, no podíamos caminar y hoy en día ya aprendimos. De tal manera que lo mismo sucede con la integración de un departamento o de una especialidad. Tan comenzada está la integración, que como muy bien dice el doctor Ramírez, el programa de enseñanza pre-graduada está totalmente integrado. Tanto así, que no hay sino la repetición que existe en toda clase normalmente de una sola materia, pero el programa está hecho en conjunto de tal manera que las repeticiones sean las menos posibles. Dentro del tiempo del mismo bloque está hecho el programa, luego si es un sólo bloqué, pues se está haciendo la integración. Tan integrado está que los alumnos van de un sitio al otro. Ahora si se trata de la enseñanza postgraduada, pues también está integrada, ya que los residentes de primer año pasan durante los seis meses que están en Ginecología, haciendo también cursos de maternidad; si están en Maternidad, deben bajar a las reuniones de Ginecología, y así sucesivamente, a través de los cuatro años. Tal vez quien hace la pregunta tendría en mente tal vez por qué todos los especialistas no se han integrado, pero eso no tiene ya relación con la enseñanza, que es el tema que estamos tratando. De tal manera que yo contestaría que la enseñanza pre y post-graduada está integrada; naturalmente que no estamos dentro de la perfección, pero es difícil que las cosas que comienzan lleguen a la perfección desde sus comienzos.

Doctor Peralta: - La realidad del tema que se trata radica en que hay cierta discrepancia entre lo que se ha planeado y lo que está ocurriendo. Muy bien concebida y organizada la reintegración; es fruto de lo que se hace en otras partes. Pero tenemos que confesar que en la práctica no opera a cabalidad. Indudablemente hay un factor topográfico, muy separados los servicios. El ideal sería de que hubiese un solo servicio. Indudablemente la antigua división ha operado desfavorablemente para esta reintegración de obstetras y ginecólogos. Todavía se vislumbra cierto celo, a veces hay la sensación en el ginecólogo de que el obstetra no sabe la ginecología, y viceversa. También hay el celo recíproco. Es- 
to se ve con frecuencia no sólo en la concepción de los problemas en ciertos certámenes, sino que repercute para que haya una reintegración completa. Por otra parte, hay que tener en cuenta el factor relacionado ya con la Universidad en sí, con la Facultad. No se puede de manera alguna seguir haciendo un tipo de docencia a base de profesores a raticos; se necesita entonces que la Facultad prescinda de factores económicos y de personalismo, que han existido, y que entonces tenga en cuenta al profesor con mayor dedicación. $\mathrm{Si}$ se complementa esto con cierta experiencia, creo que lograríamos una perfecta reintegración en materia de Obstetricia y Ginecología.

Doctor Amaya León: -Creo que está demasiado planteado el problema de la Universidad Nacional, nos gustaría oír el punto de vista del profesor Cardona, de la Universidad de Antioquia.

Doctor Cardona: -En la Universidad de Antioquia, en la Facultad tenemos un Departamento de Obstetricia y Ginecología; se está dando la docencia a los estudiantes, en este momento, por el sistema de bloques, que opera en la siguiente forma: 4 horas en la mañana dedicados a Obstetricia y 4 horas en la tarde dedicados a Ginecología. Son los mismos estudiantes, son dos cátedras independientes; como estamos en el mismo local, es entendido que los profesores de una y otra cátedra estamos en continuo contacto y al tanto para no ir a duplicar los temas. Lo que es interesante es esto, yo me formé en otra escuela, en otros tiem- pos; entonces la Obstetricia y la Ginecología eran completamente diferentes; a mí personalmente me cuesta trabajo la fusión y lo mismo he observado en Buenos Aires, allí las dos cátedras son completamente independientes. He estado haciendo el esfuerzo, por aquello de que todo el día tiene uno que contradecirse los pensamientos para ir entrando en la fusión. A los americanos les gusta mucho, a mí se me hace que a nosotros los latinos no nos gusta. Mi impresión es esta: formar buenos obstetras, formar buenos ginecólogos. Como los estudiantes están recibiendo buena enseñanza, hasta donde los medios lo permiten, en la una parte y en la otra, a mí me suena bien que el Departamento sea uno, pero que las cátedras sean independientes, con muy buenos nexos de amistad, claro está.

Allá tenemos el R1, R2, R3, pero no tenemos el R4. Estos residentes sí están al mismo tiempo formándose en las dos. De suerte que tienen que pasar por laboratorio de Ginecología, prenatal, cirugía ginecológica, sala de partos; están haciendo las dos especialidades en el curso de los tres años. Creo que si se quiere formar bien un ginecólogo, lo más interesante es la consulta externa. Puede parecer un detalle, pero a mí me parece de fundamental importancia; yo le estoy dedicando allá la mitad del tiempo de actividades.

Estamos haciendo tres bloques por año, no estoy satisfecho, ya lo he manifestado, pues afortunadamente perte- 
nezco a las directivas de la Universi$\mathrm{dad}$, porque los tres meses son uno $\mathrm{y}$ medio y es muy poco.

Doctor Amaya León: -Un profesor de la Javeriana quiere exponer sus puntos de vista para acabar así con el problema colombiano?

Doctor Jordán: —Yo sólo voy a decir algo muy corto para que el profesor Salazar lo complemente. Nosotros no hemos tenido problema con la integración, que iniciamos hace cinco años. Me da la impresión que están diciendo una cosa y haciendo otra. Dicen que no quieren integración y sin embargo están integrando a los muchachos que el día de mañana van a dirigir la cátedra. Ese problema creo que es personal, de muchos profesores antiguos que tienen dificultad en integrarse y han obstaculizado la integración. Nosotros, como somos por lo general todos jóvenes, no hemos tenido dificultad en integrarnos y hemos aprendido en parte lo que nos faltaba de la otra especialidad.

Doctor Salazar: -Como lo decía el doctor Jordán, nosotros en el Hospital de San Ignacio de la Universidad Javeriana contamos con un personal absolutamente integrado tanto en Obstetricia como en Ginecología. El problema fue fácil de solucionar, ya que todos somos de extracción de escuelas americanas. Lo que les quiero explicar es la manera como hemos afrontado este problema de la integración; los alumnos tienen un bloque semestral que se reduce a veinte semanas, de las cuales las tres primeras semanas son de enseñanza únicamente teórica. La primera semana la toma el Departamento de Ciencias Básicas de la Universidad. Reciben los estudiantes una documenración completa de embriología, de órganos genitales, especialmente de la mujer. Se apela también a los Departamentos de Fisiología, Farmacología y Genética. Las segunda y tercera semanas nosotros la dedicamos a enseñar la fisiología gineco-obstétrica; en las semanas restantes, el estudiante entra a prácticas, dedicándosele a este tipo de enseñanza el 90\% del tiempo; sólo recibe una conferencia magistral diaria. Después de la conferencia, que es lo primero del día, los estudiantes se dividen en grupos, uno de los cuales pasa a consulta externa, tres horas diarias, consulta que se alterna tres semanas en Ginecología y tres en Obstetricia por 6 semanas. El estudiante queda solo en un consultorio con un instructor. Otro grupo va durante 15 días al Instituto Nacional de Cancerología, otro queda con un instructor, generalmente para enseñanza de quirúrgica; otro grupo va al recién nacido, y un cuarto grupo pasa al servicio por tres o cuatro semanas, a desempeñar el pa pel de interno, luego pasa sus informes a corrección del residente jefe.

En cuanto a la enseñanza post-graduada, tenemos un ambicioso programa de 4 años. El primer año es el internado rotatorio. El segundo año es rotaciones por diversos servicios. El tercer año ya se encarga de lleno el residente de la atención de Obstetricia y parte de la Ginecología. El último año ya es el ginecólogo jefe y se encarga de todo el trabajo del hospital. 
Doctor Amaya León: -Como el doctor Cardona mencionó la Escuela argentina, quisiéramos oír al doctor $\mathrm{Di}$ Paola.

Doctor Di Paola: _En la Argentina las dos especialidades se enseñan en forma separada. Tres profesores de Obstetricia y dos de Ginecología. Ambas especialidades buscan una coordinación en la enseñanza mediante un intercambio de ideas y de programas entre los profesores titulares. En las sociedades científicas, ambas especialidades están integradas. De esa manera de proceder estamos satisfechos hasta ahora. Hemos resistido a la integración, porque hemos visto que en los países, que son casi todos, en donde funciona, los profesores se dedican exclusivamente a una cosa o a la otra. Creo que es difícil que una persona pueda abarcar todos los problemas que a diario se presentan en a m b a s especialidades, complicando más la tarea.

En las Escuelas de Boston y Baltimore, las que más aportes científicos han hecho en los últimos años, las dos especialidades estaban separadas. En Baltimore se integraron, y no están nada contentos con los resultados de la integración. Es conveniente, a mi modo de ver, que los jóvenes hagan ambas especialidades, tanto por razones científicas como económicas.

También hemos visto otra cosa muy interesante en los servicios integrados: No se tratan casi los problemas oncológicos; he visto llegar enfermas con carcinomas al Mount Sinai, que no atienden sino que las mandan al Me- morial. Esto no debiera ser, ya que es muy distinto tratar un carcinoma de laringe y un carcinoma de útero. Creo que esto de integración es una moda que pasará.

-De acuerdo con lo que acaba de decir el doctor Di Paola, en la Escuela del Litoral, que está en Rosario, existen una cátedra de Ginecología y dos de Obstetricia.

En cuanto al problema de pre y postgraduados tenemos solucionado el problema en esta forma: En el de pre-gracuados pensamos que deben de hacer un año; lo tenemos dividido en tres ciclos de 4 meses, que corresponden a Obstetricia y Pediatría; Cirugía general, Traumatología y Ginecología; Clínica médica e infecciosa.

El departamento de post-graduados es un proyecto en el cual únicamente se ha podido hacer un departamento, que, a cargo de las distintas cátedras, dé cursos para los post-graduados. Pero el proyecto más grande es el de las residencias, que posiblemente se inicie el año entrante. Estas serán de tres años, los médicos que vayan a hacerlas tendrán 2 años de Obstetricia y uno de Ginecología, o a la inversa. Haciendo la renovación anual de cada uno de esos integrantes. Independientemente de eso, en forma oficial, la Facultad da actualización de temas, mesas redondas y simposios, cursos a cargo de especialistas y también conferencias magistrales. Las sociedades hacen cursos de perfeccionamiento y además una cosa que omitió el doctor Di Paola, de la 
cual es el Alma Mater y que se hace todos los años en nuestro país: La Jornada Ginecológica de la FLASOG.

Doctor Avendaño: -En Chile la tradición universtiaria es muy fuerte, en el sentido de la enseñanza en cátedras separadas. Debo decir que sólo, de las tres universidades que tenemos, en la Universidad Católica se hace una enseñanza conjunta con un solo profesor. Digo que la tradición es muy fuerte porque hasta este momento no se ven indicios de que se pretenda una integración. Hay que considerar por una parte la enseñanza pre y post-grado, y por otra, la universidad y los servicios hospitalarios, ya que las universidades se extienden naturalmente a los servicios de hospital con motivo a la tendencia a la enseñanza práctica. En el nivel post-grado, podemos considerar iniciada la integración; nuestros graduados hacen tres años de residencia conjuntamente en Obstetricia y Ginecología, dividiendo el trabajo del día en $50 \%$ para cada una aproximadamente y entregándole las noches todas a Obstetricia, como es lógico. Los servicios asistenciales están todos separados, con la sola excepción de Barrofluco de Santiago. Hasta que una nueva generación venga a imponerse, nosotros no vemos la posibilidad de que se realice esta integración. Creemos que se debe llegar a ella gradualmente, pasando primero por una coordinación, que no existe en el sistema de autonomía actual.

Finalmente debo decir que estoy de acuerdo con muchos de los conceptos que ha emitido el profesor Di Paola, y a este respecto debo recordar a un viejo profesor de la Universidad de Columbia, Benjamín Watson, quien en su juventud hizo ambos entrenamientos conjuntos, Obstetricia y Ginecología, pero dice que nunca las ejerció simultáneamente. Decía que cuando era joven se había dedicado a proteger el periné, y ahora que estaba viejo, el periné lo protegía a él.

Doctor Castelazo: -Aun cuando mi formación profesional se hizo en una escuela en donde estaban totalmente separadas la enseñanza de la Ginecología y la Obstetricia, hemos luchado en México por la integración, a nivel de las primeras etapas, pre y post-graduadas, y la hemos logrado. En la actualidad los estudios pre-graduados se hacen usando Ginecología y Obstetricia en conjunto. Los estudios de post-graduado que abordan generalmente al profesionista en los primeros cinco o diez años, también se realizan en conjunto. Me parece que no se trata mucho de pareceres o de gustos; se ha mencionado ya la resistencia existente en muchas universidades porque se trata de profesores muy respetados y respetables, de gran ascendencia e inclusive de gran influencia en los gobiernos de las universidades, que por ejercer una de las dos ramas le hacen mala cara a la integración. La misma irritación que le produce al profesor $\mathrm{Di}$ Paola, p.e., el que una enferma tenga que referirse a una Institución de Cancerología en vez de tratarse en un Centro Ginecológico, nos produce a nosotros el que un médico que sabe hacer 
una aplicación de fórceps no sepa tratar un trastorno menstrual. Pensamos que la alta especialización en Ginecología o en Obstetricia puede ser ejercida por quien ha recibido una preparación integrada y completa, que abarque las dos materias; con todos los conceptos universales que unen a estas materias. Pero no nos parece que se prepare a un alumno en una de ellas con desconocimiento casi absoluto de la otra. Hay médicos que se entrenan desde el primer año en una de las dos ramas y le hacen el feo a la otra; nos parece que la base de la integración profesional de este especialista es sumamente defectuosa. Un señor que 1lega a ser un gran ginecólogo con especialización en endocrinología, debe ser una persona que ha hecho muchas aplicaciones de fórceps, que se ha desvelado muchas noches por las pacientes, que se ha enterado con intimidad, a través de un ejercicio sistematizado que incluya preparación académica, en Obstetricia. Lo mismo podemos decir de las altas especializaciones en general; no las criticamos. Como el profesor Di Paola decía, se han extendido tanto los campos en la Ginecología y la Obstetricia que no es posible abarcarla toda con la misma calidad de preparación. Pero quien opte a una super-especialización, debe conocer con profundidad los dos campos.

Este es nuestro concepto; lo ejercemos en el post-graduado en donde tenemos dependientes de la Universidad y ejercemos en el hospital cursos de adiestramiento que son de un año, residencias que son de tres años, y cur- sos para grado académico en Ginecología y Obstetricia que duran otros tres años. Una persona que ha salido después de siete años, y esto está en vigor, de estar estudiando Ginecología y Obstetricia, puede y desde muchos aspectos debe, según sus propias inclinaciones, super-especializarse en cualquier sub-rama de la Ginecología o de la Obstetricia. Está capacitada por conocimientos básicos para hacerlo, conocimientos practicados de hecho, no solamente leídos.

Doctor Figueroa: _En Honduras la integración está todavía en evolución; quizás por simple mimetismo, hemos adoptado el sistema de departamentización. No sabemos, el tiempo lo dirá, si éste será un sistema bueno. Pienso que la Obstetricia, a pesar de ser una fase transitoria en la vida ginecológica de la mujer, debe estudiarse por aparte, con la aplicación de las bases fundamentales de tipo ginecológico. Así como se ha departamentalizado la Pediatría, que no es más que la Medicina Interna, aplicada al niño, podemos también aplicar ese estado ocasional de la vida ginecológica de la mujer, llamado fase obstétrica. En Honduras tenemos que darle una importancia más grande, sin que por esto se le rebajen los méritos a la Ginecología, a los conocimientos obstétricos, sencillamente porque estamos formando médicos generales. Médicos que tienen que ejercer en el medio rural, por lo menos en el año de servicio social o de servicio rural, que le toca. En este año le toca ejercer independientemente, sin libros que consultar, colegas que le puedan ayudar. 
Tiene que decidir con la rapidez que exigen las emergencias obstétricas, que no la exigen las ginecológicas; está la vida de una mujer y de un niño de por medio; por eso nuestros médicos generales deben de tener unos conocimientos obstétricos muy profundos. Por eso sigo pensando que la Obstetricia, sin desligarse de la Ginecología por completo, debe seguirse viendo con mejores ojos y con una enseñanza más fundamental.

Doctor Lavergne: -Nuestra Escuela de Medicina en Panamá sólo cuenta con diez años de existencia; yo he estado al frente de la cátedra por espacio de tres años. Debo decir que mi opinión favorece la integración, debido a que nosotros somos el fruto de las Sociedades de Obstetricia y Ginecología originalmente, y allí tenemos el concepto intacto. Además, todos los que propulsamos esta idea, creemos que el embarazo es algo normal en la vida de la mujer y que presenta situaciones patológicas, lo mismo que la menstruación, que también es algo normal en la vida sexual de la mujer. En mi opinión la persona que se va a dedicar a la especialidad femenina debe tener conocimientos fundamentaels de ambas variedades de la misma rama. Fui formado en una escuela norteamericana, en donde el concepto unitario prevalecía a pesar de que hay maternidades especializadas, profesores especializados en un ramo de la Ginecología. Pero como nuestro medio es subdesarrollado, nuestros médicos deben ser médicos generales cuando salen de la escuela, que cuando salen a ejercer en el campo del interior de la República, deben tener conocimientos completos de ambas ramas; si bien es cierto que se pueden referir casos ginecológicos y que la obstetricia debe resolverse inmediatamente, hacemos lo posible porque ellos estén capacitados para resolver dichos problemas al salir del post-graduado.

Doctor Miranda: -En la Facultad de Medicina de la Universidad de Guayaquil las cátedras de Ginecología y Obstetricia están divididas. Me cabe el honor de ser el profesor titular de Clínica Obstétrica; voy a plantear brevemente la forma en que tenemos organizada nuestra cátedra: Tenemos tres profesores agregados y cuatro jefes de clínica. En Guayaquil la Maternidad no está ubicada dentro del Hospital General. Contamos con doscientas veinte camas; entonces sucesivamente, previo un curso teńrico que dura dos meses, hacemos qu. los estudiantes pasen a la consilta externa y a la sala de partús cuando tengan un buen adiestramiento en la semiología obstétrica. Luego no tenemos curso de post-graduado. Creo que hay un factor económico común a muchos países de Latinoamérica; el hablar de integración de clínica obstétrica y clínica ginecológica significa un gasto muy crecido. Propondría que de esta Mesa Redonda salga una recomendación para todos los representantes de las Sociedades de Obstetricia y Ginecología que pertenecen a la FLASOG, que propugnen a sus respectivas Facultades y con la anuencia de dichas Facultades el verificar cursos de postgraduados hasta que la situación económica lo permita para la Facultad misma. 
Doctor González Govea, de la Universidad Nacional del Zulia. No está.

Doctor Vidalón: -Estoy de acuerdo con muchos de los conceptos emitidos hasta ahora y con la conducta que han seguido; en el Perú, nuestra Universidad de San Fernando en Lima, que integra la de San Marcos, tiene muchísimos años y nunca se ha pensado en la integración. Son cátedras completamente independientes que inclusive funcionan en diferentes servicios, en diferentes hospitales. Se ha hablado en unas oportunidades de la integración, pero como va contra una tradición implantada, surgieron siempre ciertas dificultades; además, la idiosincrasia de nuestro pueblo tiene más objetividad, más práctica y énfasis en preparar a los estidiantes de Medicina en la Obstetricia, ya que en el territorio tan dilatado y con calidad de población tan variada, los recursos hospitalarios que apenas se están subsanando exigían de la Facultad de Medicina una preparación sobre todo en Obstetricia. Por lo menos el $75 \%$ de la población rural casi siempre ha sido atendido por comadronas, últimamente por obstetrices preparadas por la Facultad y por hombres del campo, la mayor parte curiosos o curiosas. De ahí la gran mortalidad, morbilidad que tenemos en nuestras estadísticas nacionales; es por esta razón por lo que no se ha pensado en la integración.

Hace ya aproximadamente dos años tenemos dos Facultades de Medicina que se han creado, una en el Norte y otra en el Sur, casi con caracteres y funciones de Universidades piloto. En- tiendo que la del Norte está organizada exactamente como las de los Estados Unidos. La del Sur, en Arequipa, tiene esa misma tendencia. Ambas cuentan afortunadamente con magníficos hospitales y centros de servicio modernos; es por eso por lo que yo quería hacer mención de esta nueva tendencia porque estamos en un período de cambios radicales. En la Universidad de San Fernando tuvimos una crisis grave, por los momentos dramáticos que tuvieron lugar en la Facuitad precisamente en esta época del año pasado, de los cuales ustedes seguramente están enterados, tuvimos que renunciar en masa el $95 \%$ de los profesores para conservar la autonomía. Apoyados por la opinión general, fundamos la Universidad de Ciencias Médicas Cayetano Heredia, que está funcionando hace dos meses. Con esto resulta una nueva era para la Facultad de Medicina, tenemos que adaptarnos a un nuevo curriculum, a un nuevo estado de cosas y sobran profesores.

Doctor Botero Peláez, de Colombia. No está.

Doctor Perna, de la Universidad de Cartagena: - No tengo sino un año de ser profesor de la Universidad de Cartagena, pero en mi afán de que la Universidad de Cartagena por lo menos expusiera brevemente lo que se hace en ella en materia de Ginecología y Obstetricia, quise poner mi nombre en esta Mesa Redonda. Nosotros tenemos un departamento de Ginecología y Obstetricia, integral. Creemos firmemente que es la mejor manera de encarar la 
enseñanza al estudiantado. Estamos de acuerdo con el doctor Peralta en que no creemos en profesores de una hora o de raticos. Tenemos profesores de medio tiempo o de tiempo completo, que están llevando a cabo la enseñanza en bloques pequeños. Tenemos bloques de seis a ocho alumnos que enseñamos seis semanas en Ginecología y un poco más en Obstetricia; en esta forma, por medio de una enseñanza casi individual, por medio de sesiones clí- nicas, conferencias y consulta externa, hemos visto muy satisfactoriamente los resultados de esta enseñanza, que es más práctica que teórica; por lo tanto, quiero simplemente añadir que mientras más individualmente se haga la enseñanza, no en forma de conferencias a cuarenta alumnos que no se van a volver a ver en el curso del día, sino en forma de bloques, que es la que estamos llevando a cabo, es la más adecuada para la docencia de la Ginecología. 